\title{
Detection of coherent superpositions of phase states by full counting statistics in a Bose Josephson junction
}

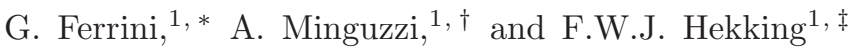 \\ ${ }^{1}$ Université Joseph Fourier, Laboratoire de Physique et Modélisation \\ des Mileux Condensés, C.N.R.S. B.P. 166, 38042 Grenoble, France
}

(Dated: November 21, 2018)

\begin{abstract}
We study a Bose Josephson junction realized with a double-well potential. We propose a strategy to observe the coherent superpositions of phase states occurring during the time evolution after a sudden rise of the barrier separating the two wells. We show that their phase content can be obtained by the full counting statistics of the spin-boson operators characterizing the junction, which could be mapped out by repeated measurements of the population imbalance after rotation of the state. This measurement can distinguish between coherent superpositions and incoherent mixtures, and can be used for a two-dimensional, tomographic reconstruction of the phase content of the state.

PACS numbers: 03.75.-b,03.75.Mn
\end{abstract}

\section{INTRODUCTION}

Superpositions of quantum states are at the heart of quantum physics. They are important for precision tests and are at the basis of quantum information and communication. The fate of these superpositions in the presence of interactions with the environment has been largely studied to understand the cross-over from a quantum to a classical description [1]. Although fragile and subject to decoherence, superposition states have been experimentally observed with trapped ions [2] and cavity photons [3].

Ultracold atomic gases are another system potentially suitable for realizing macroscopic superpositions. We are interested here in the study of superpositions of (angularmomentum) coherent states [4], which could be realized in a Bose Josephson junction made by a double-well trap. Such superposition states are predicted to form [5] during time evolution after a sudden rise of the barrier separating the two wells, starting from a coherent state at the initial time. An initially coherent state corresponds to the ground state in the regime where the tunneling energy dominates the repulsion energy, and is commonly realized in current experiments on Bose Josephson junctions [6]. The subsequent dynamical evolution after the rise of the barrier is driven by interactions only, and at specific times the system undergoes the formation of several multicomponent superpositions of phase states, finally coming back to the initial state after a revival time. In a pioneering experiment [7] on ultracold bosons in optical lattices, revivals have been indeed observed, but it was not possible to directly access the macroscopic superpositions which were formed during intermediate stages of the evolution.

Multicomponent superpositions of phase states are characterized by a sequence of equal and equidistant peaks along the equator $\theta=\pi / 2$ of the Husimi phase distribution $Q(\theta, \phi)=\langle\theta \phi|\hat{\rho}| \theta \phi\rangle$ [8, 9], with $|\theta \phi\rangle$ an angular-momentum coherent state and $\hat{\rho}$ the density ma- trix. Each peak corresponds to the contribution of a given coherent state belonging to the coherent superposition. An important issue to address is how to reveal the effect of decoherence on quantum superpositions of macroscopically distinguishable states. We proceed by analogy with the macroscopic superpositions of phase states studied in the context of photons in cavities [10]. We expect that generic sources of noise would tend first to destroy the coherence between the components of the superposition, by projecting it onto an incoherent mixture of coherent states. On a longer time scale each component is expected to relax, yielding a total spreading of the phase profile.

In this work we address the question on how to identify the quantum macroscopic superposition of phase states and how to distinguish them from mixtures. Our approach is substantially different from the one of Ref. 11] which is devoted to map out the Husimi distribution. Although the Husimi phase distribution is in one-to-one correspondence with the full density matrix [9], in practice it is almost insensitive to the difference between a coherent superposition of phase states and the corresponding incoherent mixture. This is because the Husimi distribution is the diagonal of the density matrix represented over coherent states. Figure 1 (top panel) shows the Husimi distribution for a three-component superposition of phase states and for the incoherent mixture, the tiny difference between the two being illustrated in the inset.

The paper is organized as follows. After introducing the model in Sec. II, in Sec. III we propose to reconstruct the phase distribution by mapping out all the higher order correlations, ie the full counting statistics of the spinboson operators $\hat{J}_{x}$ and $\hat{J}_{y}$ associated to the quantum model of the junction. In Sec. IV we show that it is possible to make a two-dimensional reconstruction of a Wigner-like distribution function. We conclude in Sec. $\mathrm{V}$ after discussing experimental issues. 

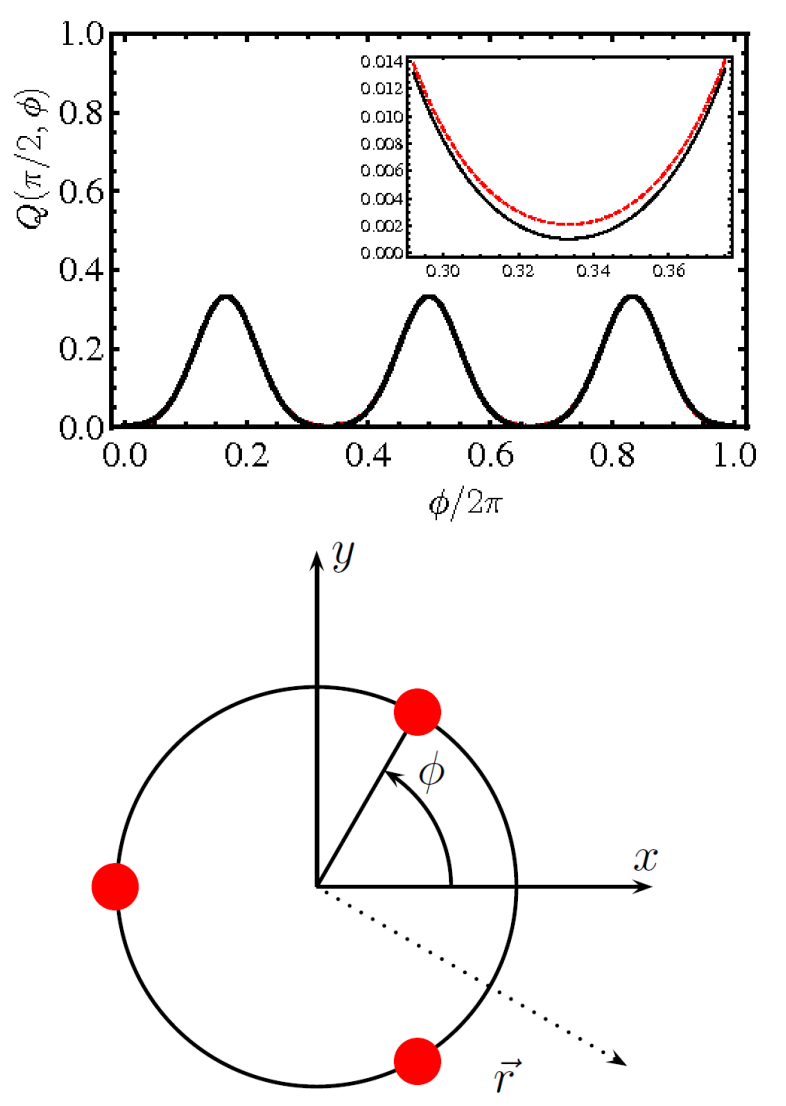

FIG. 1: (Color online) Top panel: Dimensionless Husimi distribution $Q(\theta=\pi / 2, \phi)$ for a three-component superposition of phase states (solid line) and for the corresponding incoherent mixture (dashed line), as a function of the phase $\phi / 2 \pi$ for $N=20$ particles. The inset shows a zoom of the same function around $\phi=2 \pi / 3$, illustrating the difference between the superposition state and the incoherent mixture. Bottom panel: section of the Bloch sphere on the equatorial plane $\theta=\pi / 2$, parametrized by the angle $\phi$. The dots indicate schematically the phase of the three coherent states which give rise to the superposition, and correspond to the maxima in the Husimi distribution illustrated in the top panel. The vector $\vec{r}$ defines a generic direction of the spin-boson operator for which the probability distribution is considered (see text). Our convention for the $x, y$ axes is also indicated.

\section{TWO-MODE MODEL FOR THE BOSE JOSEPHSON JUNCTION}

We describe the Bose Josephson junction in the quantum regime by using the two-mode approximation. This model applies in the limit where the chemical potential of the system is smaller than the barrier between the two wells and corresponds to neglecting higher order singleparticle levels of the double-well potential. Hence we adopt the following two-mode Bose-Hubbard Hamilto- nian:

$$
\begin{aligned}
H= & E_{1} \hat{a}_{1}^{\dagger} \hat{a}_{1}+E_{2} \hat{a}_{2}^{\dagger} \hat{a}_{2}+\frac{U_{1}}{2} \hat{a}_{1}^{\dagger} \hat{a}_{1}^{\dagger} \hat{a}_{1} \hat{a}_{1} \\
& +\frac{U_{2}}{2} \hat{a}_{2}^{\dagger} \hat{a}_{2}^{\dagger} \hat{a}_{2} \hat{a}_{2}-K\left(\hat{a}_{2}^{\dagger} \hat{a}_{1}+\hat{a}_{1}^{\dagger} \hat{a}_{2}\right),
\end{aligned}
$$

where $\hat{a}_{i}, \hat{a}_{i}^{\dagger}$ with $i=1,2$ are bosonic field operators for the bosons in each well, satisfying the usual commutation relations $\left[\hat{a}_{i}, \hat{a}_{j}^{\dagger}\right]=\delta_{i j} ; E_{i}$ are the energies of the two wells, $U_{i}>0$ are the boson-boson repulsive interactions and $K$ is the tunnel matrix element, ie the Rabi oscillation energy in the case of a non-interacting model. We then perform the transformation onto spin-boson variables, where $\hat{J}_{x}=\left(\hat{a}_{1}^{\dagger} \hat{a}_{2}+\hat{a}_{2}^{\dagger} \hat{a}_{1}\right) / 2$ is the inter-well tunneling operator, $\hat{J}_{y}=-i\left(\hat{a}_{1}^{\dagger} \hat{a}_{2}-\hat{a}_{2}^{\dagger} \hat{a}_{1}\right) / 2$ is the current operator, and $\hat{J}_{z}=\left(\hat{a}_{1}^{\dagger} \hat{a}_{1}-\hat{a}_{2}^{\dagger} \hat{a}_{2}\right) / 2=\hat{n}$ is the population imbalance operator. The Hamiltonian is readily rewritten as

$$
\hat{H}=U_{s}\left(\hat{J}_{z}-n_{0}\right)^{2} / 2-2 K \hat{J}_{x},
$$

with $U_{s}=\left(U_{1}+U_{2}\right)$ and $n_{0}$ is related to the imbalance between the wells according to $n_{0}=-\left[E_{1}+U_{1}(N-\right.$ 1) $\left./ 2-E_{2}-U_{2}(N-1) / 2\right] / U_{s}$ [8]. In the quasi-classical limit $K N \gg U_{s}$, the angular-momentum coherent states $\left|\theta_{0} \phi_{0}\right\rangle=\sum_{m=-N / 2}^{N / 2}\left(\begin{array}{c}N \\ m+N / 2\end{array}\right)^{1 / 2} \frac{\alpha^{m+N / 2}}{\left(1+|\alpha|^{2}\right)^{N / 2}}|m\rangle$ with $\alpha=\tan \left(\theta_{0} / 2\right) \exp \left(-i \phi_{0}\right)$ are useful to describe the state of the system. In particular, the ground state corresponds to the coherent state with $\theta_{0}=\pi / 2$ and $\phi_{0}=0$.

In the following we consider the time evolution of the Hamiltonian (2) after a sudden rise of the barrier, namely we set $K=0$, starting from the noninteracting ground state $\left|\theta_{0}=\pi / 2, \phi_{0}=0\right\rangle$ as the initial state. Such a dynamical evolution occurs entirely in the equatorial plane of the Bloch sphere as $\hat{J}_{z}$ is a constant of the motion. We focus on the specific time $T_{q}=2 \pi /\left(U_{s} q\right)$ where a coherent superposition of $q$ coherent states is formed, according to

$$
\left|\psi_{q}\right\rangle=\tilde{u}_{0} \sum_{k=0}^{q-1} \tilde{c}_{k}\left|e^{-i \frac{2 \pi k}{q}-i \frac{\pi}{q}}\right\rangle
$$

with $\tilde{u}_{0}=\frac{1}{q} e^{\frac{i \pi N}{2 q}} \sum_{m=0}^{q-1} e^{-i \frac{\pi m(m-1)}{q}}$ and $\tilde{c}_{k}=e^{i \frac{\pi k(N+k+1)}{q}}$; we have assumed $q$ odd. Our aim is to reveal the phase content of such a state. For this purpose we consider the operators $\hat{J}_{x}$ and $\hat{J}_{y}$ which have an action close to that of the relative-phase operators $\cos \phi$ and $\sin \phi$. However, the latter act incorrectly on the boundary states $| \pm N / 2\rangle$. The average over a coherent state characterized by a relative phase $\phi_{0}$ and population imbalance $n$ gives $\left\langle\alpha\left|\hat{J}_{x}\right| \alpha\right\rangle=n \cos \phi_{0}$ and $\left\langle\alpha\left|\hat{J}_{y}\right| \alpha\right\rangle=n \sin \phi_{0}$. While the momentum distribution accesses only the first-moment averages $\langle\hat{\cos } \phi\rangle,\langle\sin \phi\rangle$ [12], in order to access the entire phase distribution we need to consider all the higher correlations. 


\section{FULL COUNTING STATISTICS OF THE ANGULAR-MOMENTUM OPERATORS}

The probability distribution of the eigenvalues of an observable contains the information equivalent to the knowledge of all the moments of the distribution itself. In turn, the latter are known once the generating function is known, according to a full counting statistic approach [13].

Let us consider a spin-boson operator $\hat{J}_{r}$ in the direction specified by the two-dimensional vector $\vec{r}=$ $(\sin \phi,-\cos \phi)$ in the $(x, y)$ plane. The generating function for the probability distribution of the eigenvalues $r$ that correspond to the eigenstates $|r\rangle, \hat{J}_{r}|r\rangle=r|r\rangle$, is given by

$$
h_{\phi}(\eta)=\left\langle e^{-i \eta \hat{J}_{r}}\right\rangle
$$

Here, $\hat{J}_{r}=\hat{J}_{x} \sin \phi-\hat{J}_{y} \cos \phi$ and $\langle\ldots\rangle$ indicates the quantum average over the state of the system. Considering for simplicity a pure state $|\psi\rangle$ and expanding in terms of the eigenstates $|r\rangle$ of $\hat{J}_{r}$ we have

$$
h_{\phi}(\eta)=\sum_{r} e^{-i \eta r}|\langle\psi \mid r\rangle|^{2}
$$

where the eigenvalue $r$ takes integer values in the interval $[-N / 2, N / 2]$ and we have assumed $N$ to be even. We shall denote $P_{\phi}(r)=|\langle\psi \mid r\rangle|^{2}$ the corresponding probability distribution (for a generic density matrix $\hat{\rho}$, $\left.P_{\phi}^{\hat{\rho}}(r)=\operatorname{Tr}(\hat{\rho}|r\rangle\langle r|)\right)$. Its shape reflects the phase content of the state projected along the direction specified by the vector $\vec{r}$ (see the bottom panel of Fig 1 for a sketch). As we will detail in Sec.V, this could be accessed experimentally.

In order to determine the probability distribution $P_{\phi}(r)$, we evaluate the generating function Eq. (4) analytically both for coherent superpositions and mixtures. Specifically, we focus on the three-component superposition of phase states and on the corresponding incoherent mixture of the same three equally-weighted phase states. We use the definition of angular-momentum coherent states and the disentangling formula [4]

$$
e^{-i \eta \hat{J}_{r}}=e^{-\tau^{*} \hat{J}_{-}} e^{-\log \left(1+|\tau|^{2}\right) \hat{J}_{z}} e^{\tau \hat{J}_{+}},
$$

with $\tau=\tan (\eta / 2) e^{-i \phi}$. We thus obtain the final results for the generating functions:

$$
\begin{aligned}
& h_{\phi}^{\text {mixt }}(\eta)=\left|\tilde{u}_{0}\right|^{2} \sum_{k=0}^{q-1} \\
& \left\{\left|\cos \frac{\eta}{2}\right|+i \sin \frac{\eta}{2} \operatorname{sign}\left[\cos \frac{\eta}{2}\right] \sin \left(\frac{2 \pi k}{q}+\frac{\pi}{q}-\phi\right)\right\}^{N}
\end{aligned}
$$

for the incoherent mixture and

$$
\begin{aligned}
& h_{\phi}^{c o h}(\eta)=h_{\phi}^{\text {mixt }}(\eta) \\
& +\left|\tilde{u}_{0}\right|^{2} \sum_{k \neq k^{\prime}=0}^{q-1} \frac{\tilde{c}_{k} \tilde{c}_{k^{\prime}}^{*}}{2^{N}}\left\{\left|\cos \frac{\eta}{2}\right|\left(1+e^{-i \frac{2 \pi\left(k-k^{\prime}\right)}{q}}\right)\right. \\
& \left.+\sin \frac{\eta}{2} \operatorname{sign}\left[\cos \frac{\eta}{2}\right]\left(e^{i\left(\frac{2 \pi k^{\prime}}{q}+\frac{\pi}{q}-\phi\right)}-e^{-i\left(\frac{2 \pi k}{q}+\frac{\pi}{q}-\phi\right)}\right)\right\}^{N}
\end{aligned}
$$

for the coherent superposition. The probability distribution $P_{\phi}(r)$ is readily obtained by Fourier transforming the above expressions.

By considering the projections along the $x$ and $y$ directions, respectively, we obtain the full counting statistics of the operators $\hat{J}_{x}$ and $\hat{J}_{y}$. This is illustrated in Fig 2 for the three-component superposition $\left|\psi_{3}\right\rangle$ of phase states as well as for the corresponding mixture. The distribution is peaked around the semiclassical values for $\left\langle\hat{J}_{x}\right\rangle=(N / 2) \cos ( \pm \pi / 3),(N / 2) \cos (\pi)$ and $\left\langle\hat{J}_{y}\right\rangle=$ $(N / 2) \sin ( \pm \pi / 3),(N / 2) \sin (\pi)$. We find that the distribution $P_{\pi / 2}(r)$ displays a noticeable difference between the mixture and the coherent superposition: the latter displays oscillations which are absent in the former. The presence of fringes in the distribution of the eigenvalues of angular momentum operators for superposition states was also noticed in the context of the dynamics of the quantum non-linear rotator by Sanders [14]. The function $P_{\pi}(r)$ instead does not display fringes for the threecomponent superposition because its components do not overlap when projected along the $y$-direction (see Fig 1, bottom panel); as a result no interference effect takes place in this case.

The result for the three-component state extends to higher-component superpositions; the two-component one instead cannot be distinguished from the corresponding incoherent mixture by this method, due to the specific form of its state components. The full counting statistics of the operator $\hat{J}_{z}$ could also be defined, but does not yield any useful information about the considered superpositions of phase states as it coincides with the binomial distribution $P_{\phi=0}(r)=\frac{1}{2^{N}}\left(\begin{array}{c}N \\ \frac{N}{2}+r\end{array}\right)$ of the initial coherent state.

\section{TWO-DIMENSIONAL RECONSTRUCTION OF THE PHASE DISTRIBUTION}

A two-dimensional (2D) tomographic reconstruction of the phase content of a state in the $(x, y)$ plane is possible, using the concept of the Radon transform [15]. This has been also used for coherent superpositions with cavity photons 3 . The $2 \mathrm{D}$ distribution function $f(x, y)$ is obtained once all the one-dimensional projections $P_{\phi}(r)$ are known as a function of $\phi$ in the interval $[0,2 \pi]$. One 

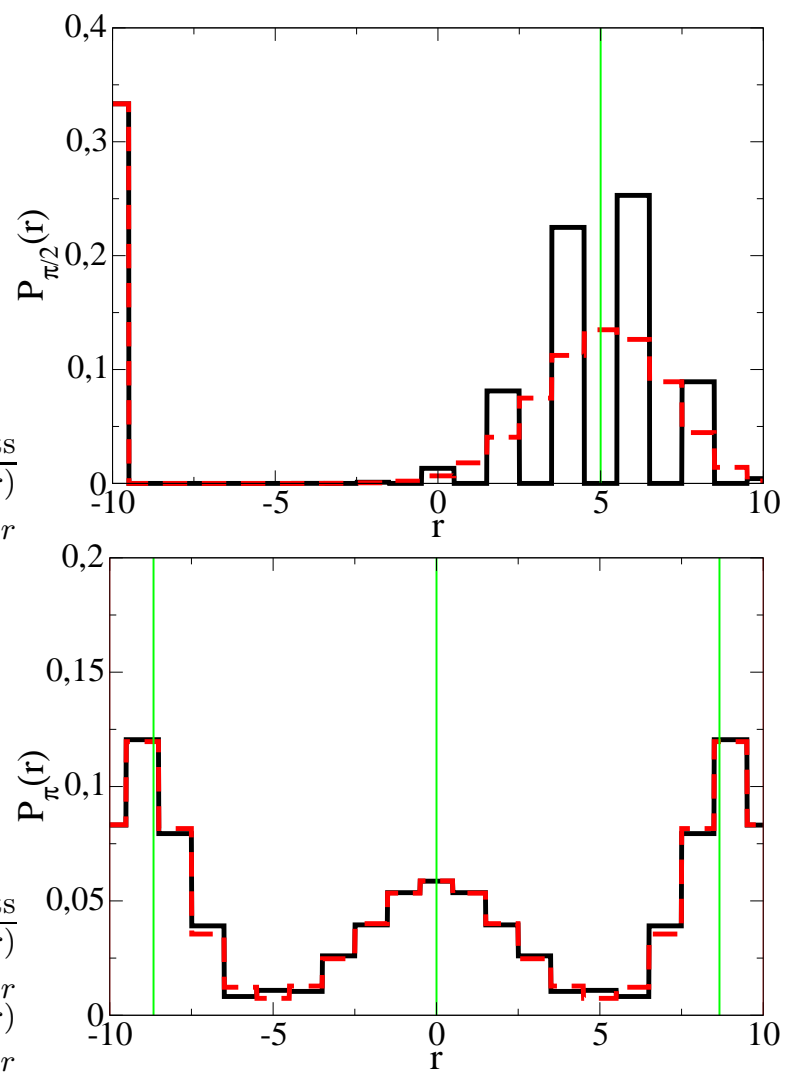

FIG. 2: (Color online) Eigenvalue distribution $P_{\phi}(r)$ corresponding to $\hat{J}_{x}(\phi=\pi / 2)$ and $\hat{J}_{y}(\phi=\pi)$ for the threecomponent coherent superposition (solid lines) as well as for the incoherent mixture of the same phase states (dashed lines) with $N=20$. The vertical lines correspond to the semiclassical values for $\left\langle\hat{J}_{x}\right\rangle$ and $\left\langle\hat{J}_{y}\right\rangle$ for the coherent states entering the superposition.

should invert the expression

$$
P_{\phi}(r)=\int_{-\infty}^{+\infty} d x \int_{-\infty}^{+\infty} d y f(x, y) \delta(r-x \sin \phi+y \cos \phi),
$$

which, using the definition of the generating function in Eq. (5), yields as a final result

$$
f(x, y)=\frac{1}{(2 \pi)^{2}} \int_{0}^{\pi} \eta d \eta \int_{0}^{2 \pi} d \phi h_{\phi}(\eta) e^{i \eta(x \sin \phi-y \cos \phi)} .
$$

This is a quasi-probability distribution for the noncommuting operators $J_{x}$ and $J_{y}$. It could be regarded as the two-dimensional projection on the equatorial plane of the Bloch sphere of a $\mathrm{SU}(2)$ Wigner function in the spirit of Ref.[17]. It is readily verified that the marginal probability distribution obtained by integration of $f(x, y)$ along one of the two directions $y$ or $x$ yields the onedimensional distributions $P_{\phi}(r)$ with $\phi=\pi / 2$ and $\pi$, respectively. The function $f(x, y)$ is not in one-to-one correspondence with the state of the system. However, for the specific superpositions of phase states which we consider here it yields the main information about the phase structure of the state. Furthermore, in the case of a coherent superposition of phase states it is markedly different from the corresponding incoherent mixture.

Figure 3 illustrates the 2D quasi-probability distribution $f(x, y)$ for a three component coherent superposition. It shows three pronounced maxima in correspondence with the three coherent states giving rise to the macroscopic superposition. It also displays oscillations between the maxima, due to interferences between the components. The 2D quasi-probability function evaluated for the corresponding incoherent mixture also exhibits the main peaks but the fringes are strongly suppressed (see Fig 3 bottom panel), the small remaining oscillations being intrinsically due to the definition of the function $f(x, y)$ as a Fourier transform in angular variables.

\section{EXPERIMENTAL ISSUES AND CONCLUDING REMARKS}

For each choice of the angle $\phi$ the probability distribution $P_{\phi}(r)$ can be experimentally accessed by repeated measurements of the corresponding angular momentum operator $\hat{J}_{r}$. Indeed, since the eigenstates of $\hat{J}_{r}$ form an orthonormal basis, each superposition state decomposes as $\left|\psi_{q}\right\rangle=\sum_{r=-N / 2}^{N / 2} c_{r}^{q}|r\rangle$ with $c_{r}^{q}=\left\langle r \mid \psi_{q}\right\rangle$. Then, according to the postulates of quantum mechanics, after a (projective) measurement of $\hat{J}_{r}$ the state jumps to the state $|r\rangle$ with probability $P_{\phi}^{c o h}(r)=\left|c_{r}^{q}\right|^{2}$, and the corresponding outcome of the measurement is $r$. The full distribution $P_{\phi}(r)$ is obtained by repeating this procedure many times, each time preparing the system in the same initial state [18]. The measurement of $\hat{J}_{r}$ for a generic angle $\phi$ can be achieved by measuring the population imbalance $\hat{J}_{z}$ between the two wells - a variable typically accessed in experiments [6] - after proper rotations of the state over the Bloch sphere 20]. More precisely, measuring $\hat{J}_{r}$ on the quantum state $|\psi\rangle$ is achieved by measuring $\hat{J}_{z}$ on the rotated state $e^{i \pi J_{x} / 2} e^{i \phi J_{z}}|\psi\rangle$. Each rotation can be implemented by a time evolution of the system with Hamiltonian (2) once the interaction strength $U_{s}$ is tuned to zero (eg by a Feshbach resonance). The rotation direction and angle can be chosen by tuning the height of the barrier, the energy bias between the wells and the time-evolution interval [21].

In summary, using the concepts of full counting statistics we propose a method to access to the full phase content of a macroscopic superposition of phase states, as well as to reconstruct tomographically its twodimensional quasi-distribution. This method is capable to distinguish between such states and an incoherent mixture as the latter does not display fringes in the probabil- 

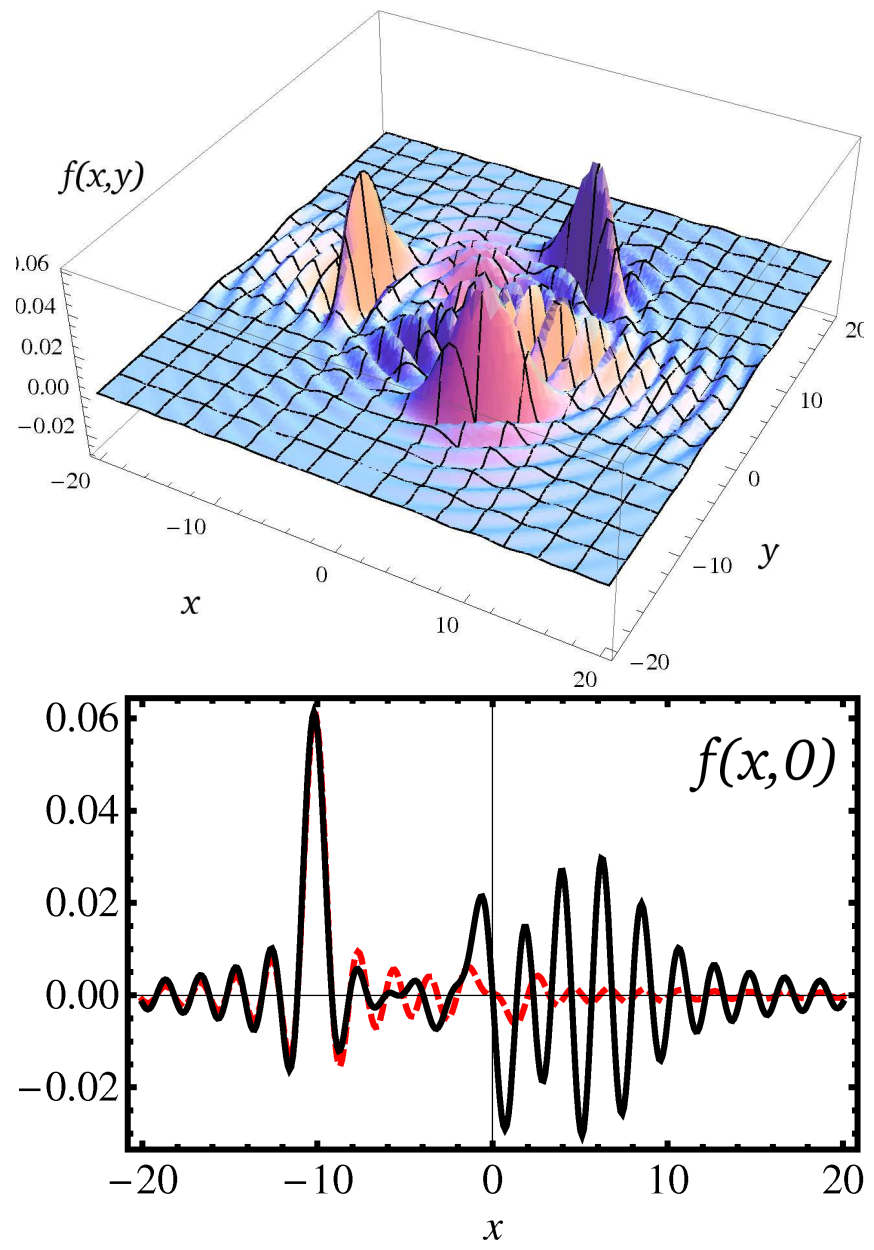

FIG. 3: (Color online) Top panel: Dimensionless 2D quasiprobability distribution $f(x, y)$ in the $(x, y)$ plane (dimensionless) for a three-component coherent superposition with $N=20$ particles. Bottom panel: section of the same quasiprobability distribution (solid line) in the same units, in the direction $y=0$. The dashed line represents the quasiprobability function $f(x, 0)$ for the corresponding incoherent mixture of the same three coherent states.

ity distribution. The superposition of phase states could be realized with current experiments on Bose Josephson junctions.

Feasibility of the current proposal requires control of the atom number on the experiment; atom losses are predicted to destroy the superposition and seem to present the major experimental challenge.

During the preparation of the present manuscript we have became aware of similar results for the 'NOON' state by Haigh et al. [22].

\section{ACKNOWLEDGMENTS}

We thank P. DeGiovanni, F. Faure, F. Piazza, A. Smerzi, D. Spenher, T. Roscilde for useful discussions.
We acknowledge financial support from CNRS and from the European MIDAS project.

* Electronic address: giulia.ferrini@grenoble.cnrs.fr

$\dagger$ Electronic address: anna.minguzzi@grenoble.cnrs.fr

¥ Electronic address: frank.hekking@grenoble.cnrs.fr

[1] W. H. Zurek, Rev. Mod. Phys. 75, 715 (2003).

[2] D.Leibfried et al., Nature 438, 639 (2005)

[3] S.Deleglise et al., Nature 455, 510 (2008)

[4] F.T. Arecchi et al., Phys. Rev. A 6, 2211 (1972).

[5] B. Yurke and D. Stoler, Phys. Rev. Lett. 57, 13 (1986)

[6] M. Albiez et al., Phys. Rev. Lett. 95, 010402 (2005); Y. Shin et al., Phys. Rev. Lett. 95, 170402 (2005); S. Levy et al., Nature 449, 579 (2007).

[7] M. Greiner et al., Nature 419, 51 (2002).

[8] G.Ferrini, A.Minguzzi and F.W.J.Hekking, Phys. Rev. A 78, 023606 (2008).

[9] W.M.Zhang, D.H.Feng and R. Gilmore, Rev. Mod. Phys. 62, 867 (1990).

[10] S.Haroche and J.M.Raymond, Exploring the quantum, Oxford Graduate Texts (2006).

[11] F.Piazza, L.Pezzé and A.Smerzi, Phys. Rev. A 78, 051601 (2008).

[12] L.Pitaevskii and S.Stringari, Phys. Rev. Lett. 87, 180402 (2001).

[13] L.E.Reichl, A modern course in Statistical Physics, Cambridge University Press (1984).

[14] B.C. Sanders, Phys. Rev. A 40, 2417 (1989).

[15] F.Natterer, The Mathematics of Computerized Tomography, Wiley-Chichester (1986).

[16] K. Vogel and H. Risken, Phys. Rev. A 40, 2847 (1989).

[17] S.M.Chumakov, A.Frank and K.B.Wolf, Phys. Rev. A 60, 1817 (1999).

[18] In typical experiments the system is destroyed after a measurement of $\hat{J}_{r}$. Therefore, one should model the process by a Positive Operator Valued Measure (POVM) rather than by a projective measurement [19]. However, here we are only interested in the outcomes $r$. These are predicted to be the same for both POVM and projective measurement, even if the latter does not describe properly the state of the system after the measurement.

[19] M.A.Nielsen and I.L.Chuang, Quantum computation and quantum information, Cambridge University Press (2000).

[20] T.Kim et al., Phys. Rev. A 57, 4004 (1998).

[21] Y.P.Huang and M.G.Moore, Phys. Rev. Lett. 100, 250406 (2008)

[22] T.J. Haigh, A.J. Ferris and M.K. Olsen, arXiv:0907.1333. 